\title{
Wiener-Hopf equation technique for solving equilibrium problems and variational inequalities and fixed points of a nonexpansive mapping
}

\author{
Yuehu Wang ${ }^{1 *}$ and Congjun Zhang ${ }^{2}$
}

"Correspondence:
wangyuehu8610@126.com
1School of Mathematical Science,
Anhui University, Hefei, 230601,
P.R. China
Full list of author information is
available at the end of the article

\section{Correspondence:}

1 School of Mathematical Science,

P.R. China

available at the end of the article

\begin{abstract}
In this paper, we introduce some new iterative schemes based on the Wiener-Hopf equation technique and auxiliary principle for finding common elements of the set of solutions of equilibrium problems, the set of fixed points of a nonexpansive mapping and the set of solutions of a variational inequality. Several strong convergence results for the sequences generated by these iterative schemes are established in Hilbert spaces. As the generation, we also consider two generalized variational inequalities, and obtain some iterative schemes and the proposed strong convergence theorems for solving these generalized variational inequalities, equilibrium problems, and a nonexpansive mapping. Our results and proof are new, and they extend the corresponding results of Verma (Appl. Math. Lett. 10:107-109, 1997), Wu and Li (4th International Congress on Image and Signal Processing, pp. 2802-2805, 2011), and Noor and Huang (Appl. Math. Comput. 191:504-510, 2007).
\end{abstract}

Keywords: equilibrium problems; algorithms; Wiener-Hopf equation technique; auxiliary principle

\section{Introduction}

Let $H$ be a real Hilbert space, whose inner product and norm are denoted by $\langle\cdot, \cdot\rangle$ and $\|\cdot\|$, respectively. Let $K$ be a nonempty closed convex subset of $H$. Let $T, S: K \rightarrow K$ be nonlinear mappings. Let $M: H \rightarrow 2^{H}$ be a multi-valued operator and let $f: K \times K \rightarrow R$ be a bifunction, where $R$ is the set of real numbers. Let $P_{K}$ be the projection of $H$ onto the closed convex set $K$ and $Q_{K}=I-P_{K}$, where the $I$ is the identity operator.

In 1994, Blum and Oettli (see [1]) introduced the equilibrium problem (EP) which is to find $\bar{x} \in K$, such that

$$
f(\bar{x}, y) \geq 0, \quad \forall y \in K
$$

The set of solutions of (1.1) is denoted by $\operatorname{EP}(f)$. Let $f(x, y)=\langle T x, y-x\rangle$ for all $x, y \in K$, then the EP reduces to the variational inequality problem (VIP) which is to find $\bar{x} \in K$ such that

$$
\langle T \bar{x}, y-x\rangle \geq 0, \quad \forall y \in K .
$$

O2014 Wang and Zhang; licensee Springer. This is an Open Access article distributed under the terms of the Creative Commons Attribution License (http://creativecommons.org/licenses/by/2.0), which permits unrestricted use, distribution, and reproduction in any medium, provided the original work is properly cited. 
This problem was introduced by Stampacchia (see [2]) in 1964. Related to EP and VIP, fixed point problems (FP) of a nonexpansive mapping are also considered by many authors. Recall that a mapping $S$ is nonexpansive if $\|S x-S y\| \leq\|x-y\|$ for all $x, y \in K$. Let us denote the set of fixed points of $S$ and set of solutions of problem (1.2) by $F(S), \operatorname{VI}(K, T)$, respectively.

Recently, for solving the EP, VIP, and FP, many authors have introduced and extended lots of iterative schemes.

For solving variational inequality problems:

In 1991, Shi (see [3]) demonstrated the equivalence between the VIP: $\langle T u-f, v-u\rangle \geq 0$, $\forall v \in K$ and Wiener-Hopf equation: $\left(T P_{K}+Q_{K}\right) v=f$, where $f \in H$. Noor (see [4]) established the equivalence between the generalized VIP: $\langle T u, g(v)-g(u)\rangle \geq 0, \forall g(v) \in K$ and the generalized Wiener-Hopf equation: $T^{-1} P_{K} z+\rho^{-1} Q_{K} z=0$ and introduced an iterative scheme based on this equivalence,

$$
\left\{\begin{array}{l}
g\left(u_{n}\right)=P_{K} z_{n} \\
z_{n+1}=g\left(u_{n}\right)-\rho T u_{n}
\end{array}\right.
$$

where $g^{-1}$ exists.

Afterwards, by using different generalized Wiener-Hopf equation techniques, Verma and Al-Shemas et al. introduced several algorithms for solving generalized VIPs, respectively (see, for example, [5-7]). It has been shown that the Wiener-Hopf equation techniques are more flexible and general than projection methods.

On the other hand, for getting the unified approach to solve EP, VIP and FP, many authors also suggest and analyze lots of iterative schemes for common elements of $F(S), \operatorname{EP}(f)$, $\mathrm{VI}(K, T)$.

For solving $\mathrm{EP}(f) \cap F(S)$ :

Takahashi and Takahashi (see [8]) introduced the following iterative schemes based on the viscosity approximation method:

$$
\left\{\begin{array}{l}
F\left(u_{n}, y\right)+\frac{1}{r_{n}}\left\langle y-u_{n}, u_{n}-x_{n}\right\rangle \geq 0, \quad \forall y \in K, \\
x_{n+1}=\alpha_{n} f\left(x_{n}\right)+\left(1-\alpha_{n}\right) S u_{n} .
\end{array}\right.
$$

For solving $\mathrm{EP}(f) \cap \mathrm{VI}(K, T)$ :

$\mathrm{Li}$ and $\mathrm{Su}$ (see [9]) introduced the following iterative schemes:

$$
\left\{\begin{array}{l}
y_{n}=\Pi_{C} J^{-1}\left(J x_{n}-r_{n} A x_{n}\right), \\
u_{n} \in C: f\left(u_{n}, y\right)+\frac{1}{r_{n}}\left\langle y-u_{n}, J u_{n}-J y_{n}\right\rangle \geq 0, \quad \forall y \in K, \\
C_{n+1}=\left\{z \in C_{n}: \varphi\left(z, u_{n}\right) \leq \varphi\left(z, x_{n}\right)\right\}, \\
x_{n+1}=\Pi_{C_{n+1}} x_{0} .
\end{array}\right.
$$

For solving $\mathrm{EP}(f) \cap \operatorname{VIP}(K, T) \cap F(S)$ :

Plubtieng and Punpaeng (see [10]) introduced the following iterative schemes:

$$
\left\{\begin{array}{l}
f\left(u_{n}, y\right)+\frac{1}{r_{n}}\left\langle y-u_{n}, u_{n}-x_{n}\right\rangle \geq 0, \quad \forall y \in K, \\
y_{n}=P_{C}\left(u_{n}-\lambda_{n} A u_{n}\right), \\
x_{n+1}=\alpha_{n} x_{1}+\beta_{n} x_{n}+\gamma_{n} S P_{C}\left(y_{n}-\lambda_{n} A y_{n}\right) .
\end{array}\right.
$$


For more algorithms, please see, for instance, [1-33] and the references therein.

Summarizing the above algorithms, we know that these papers have mainly used the auxiliary principle, the projection technique and iterative schemes of fixed points. However, the Wiener-Hopf equation technique which is more flexible and general than projection methods has not been used for solving $\operatorname{EP}(f) \cap F(S), \operatorname{EP}(f) \cap \operatorname{VI}(K, T)$, and $\operatorname{EP}(f) \cap$ $\mathrm{VI}(K, T) \cap F(S)$.

Remark 1.1 It is worth mentioning that although there are some papers which have applied the Wiener-Hopf equation technique to solve VIP and $\operatorname{VI}(K, T) \cap F(S)$, their research does not include $\operatorname{EP}(f)$. However, our idea is just to apply the Wiener-Hopf equation technique to study the common element problem which is related to $\operatorname{EP}(f)$.

In this paper, motivated and inspired by the above analysis and ongoing research in this field, we combine the Wiener-Hopf equation technique and auxiliary principle to introduce some iterative schemes for solving the common element problem which is related to $\operatorname{EP}(f)$. This paper is organized as follows: In Section 2, some preliminaries are presented. Section 3 is devoted to solving the $\operatorname{EP}(f) \cap \operatorname{VI}(K, T)$. In Section 4, we consider a nonexpansive mapping $S$ and obtain some iterative schemes and strong convergent results for solving $\operatorname{EP}(f) \cap \operatorname{VI}(K, T) \cap F(S)$. In Section 5, we extend the VIPs in Section 3 and Section 4 , and we get some iterative schemes and strong convergent theorems for solving $\operatorname{GVI}(K, T) \cap \operatorname{EP}(f)$ and $\operatorname{GVI}(K, T) \cap \operatorname{EP}(f) \cap F(S)$, respectively. Our results extend the corresponding results of Verma (see [6]), Wu and Li (see [29]), and Noor and Huang (see [33]).

\section{Preliminaries}

In the rest of this paper, let $H$ be a real Hilbert space, whose inner product and norm are denoted by $\langle\cdot, \cdot\rangle$ and $\|\cdot\|$, respectively. Let $K$ be a nonempty closed convex subset of $H$. Let $T, S: K \rightarrow K$ be nonlinear mapping. Let $M: H \rightarrow 2^{H}$ be a multi-valued operator and let $f: K \times K \rightarrow R$ be a bifunction, where $R$ is the set of real numbers. Let $P_{K}$ be the projection of $H$ onto the closed convex set $K$ and $Q_{K}=I-P_{K}$, where $I$ is the identity operator.

Definition 2.1 The operator $T: K \rightarrow K$ is said to be:

(i) $\mu$-Lipschitz continuous, if there exists a constant $\mu>0$ such that $\|T x-T y\| \leq \mu\|x-y\|$ for all $x, y \in K$

(ii) $r$-strongly monotone, if there exists a constant $r>0$ such that $\langle T x-T y, x-y\rangle \geq r\|x-y\|^{2}$ for all $x, y \in K$

(iii) $\gamma$-co-coercive, if there exists a constant $\gamma>0$ such that $\langle T x-T y, x-y\rangle \geq \gamma\|T x-T y\|^{2}$ for all $x, y \in K$;

(iv) relaxed $\gamma$-co-coercive, if there exists a constant $\gamma>0$ such that $\langle T x-T y, x-y\rangle \geq-\gamma\|T x-T y\|^{2}$ for all $x, y \in K$

(v) relaxed $(\gamma, r)$-co-coercive, if there exist two constants $\gamma, r>0$ such that $\langle T x-T y, x-y\rangle \geq-\gamma\|T x-T y\|^{2}+r\|x-y\|^{2}$ for all $x, y \in K$.

Definition 2.2 The multi-valued operator $M: H \rightarrow 2^{H}$ is said to be:

(i) a relaxed monotone operator, if there exists a constant $k>0$ such that $\left\langle w_{1}-w_{2}, u-v\right\rangle \geq-k\|u-v\|^{2}, \forall w_{1} \in M u, \forall w_{2} \in M v ;$ 
(ii) Lipschitz continuous if there exists a constant $\lambda>0$ such that $\left\|w_{1}-w_{2}\right\| \leq \lambda\|u-v\|$, $\forall w_{1} \in M u, \forall w_{2} \in M v$.

Lemma 2.1 (see [10]) Let the bifunction $f: K \times K \rightarrow R$ satisfy the following conditions:

(i) $f(x, x)=0$ for all $x \in K$;

(ii) $f$ is monotone, i.e. $f(x, y)+f(y, x) \leq 0$ for all $x, y \in K$;

(iii) for each $x, y, z \in K, \lim _{t \rightarrow 0} f(t z+(1-t) x, y) \leq f(x, y)$;

(iv) for each $x \in K, f(x, \cdot)$ is convex and lower semicontinuous.

Then $\operatorname{EP}(f) \neq \emptyset$.

Lemma 2.2 (see [10]) Let $r>0, x \in H$, and $f$ satisfy the conditions (i)-(iv) in Lemma 2.1. Then there exists $z \in K$ such that $f(z, y)+\frac{1}{r}\langle y-z, z-x\rangle \geq 0, \forall y \in K$.

Lemma 2.3 (see [10]) Let $r>0, x \in H$, and $f$ satisfy the conditions (i)-(iv) in Lemma 2.1. Define a mapping $T_{r}: H \rightarrow K$ as $T_{r}(x)=\left\{z \in K: f(z, y)+\frac{1}{r} \cdot\langle y-z, z-x\rangle \geq 0, \forall y \in K\right\}$.

Then the following hold:

(a) $T_{r}$ is single-valued;

(b) $T_{r}$ is firmly nonexpansive, i.e. $\left\|T_{r} x-T_{r} y\right\| \leq\left\langle T_{r} x-T_{r} y, x-y\right\rangle$ for all $x, y \in H$;

(c) $\operatorname{EP}(f)=F\left(T_{r}\right)$, where $F\left(T_{r}\right)$ denotes the sets of fixed point of $T_{r}$;

(d) $\mathrm{EP}(f)$ is closed and convex.

In [4], Noor introduced the following generalized Wiener-Hopf equation:

$$
\operatorname{Tg}^{-1} P_{K} z+\rho^{-1} Q_{K} z=0
$$

Here he assumed $g^{-1}$ exists, and note that if $g=I$, the identity operator, then (2.1) reduces to

$$
T P_{K} z+\rho^{-1} Q_{K} z=0
$$

which was introduced by Shi (see [3]). Denote the sets of solutions of (2.1) and (2.2) by $\operatorname{WHE}(T, g)$ and $\operatorname{WHE}(T)$, respectively.

Lemma 2.4 (see [3]) The variational inequality (1.2) has a solution $\bar{x} \in H$ if and only if the Wiener-Hopf equation (2.2) has a solution $\bar{z} \in H$, where $\bar{x}=P_{K} \bar{z}, \bar{z}=\bar{x}-\rho T \bar{x}$.

In 1988, Noor (see [19]) introduced the generalized variational inequality (GVIP) which is to find $\bar{x} \in H$ such that $g(\bar{x}) \in H$ and

$$
\langle T \bar{x}, g(y)-g(\bar{x})| \geq 0, \quad \forall g(y) \in K
$$

Denote the set of solutions of $(2.3)$ by $\operatorname{GVI}(K, T)$. Clearly, if $g=I$, the identity operator, the GVIP (2.3) reduces to VIP (1.2).

Lemma 2.5 (see [4]) The variational inequality (2.3) has a solution $\tilde{x} \in H$ if and only if the Wiener-Hopf equation (2.1) has a solution $\tilde{z} \in H$, where $g(\bar{x})=P_{K} \tilde{z}, \tilde{z}=g(\bar{x})-\rho T \bar{x}$, and $\rho>0$ is a constant. 
For finding the common element of the set of fixed points of a nonexpansive mapping and the set of solution of the variational inequality, Noor and Huang [33] introduced the Wiener-Hopf equation which included a nonexpansive mapping:

$$
T S P_{K} z+\rho^{-1} Q_{K} z=0
$$

Furthermore the equivalence was established between the Wiener-Hopf equation (2.4) and the variational inequality (1.2) as follows.

Lemma 2.6 (see [33]) The variational inequality (1.2) has a solution $\tilde{x}$ if and only if the Wiener-Hopf equation (2.4) has a solution $\tilde{z}$, where $\tilde{z}=\tilde{x}-\rho T \tilde{x}, \tilde{x}=S P_{K} \tilde{z}$.

Wu and $\mathrm{Li}$ (see [29]) introduced the Wiener-Hopf equation which includes a nonexpansive mapping $S$ :

$$
T S P_{K} z+w+\rho^{-1} Q_{K} z=0, \quad \forall w \in M S P_{K} z
$$

and established the equivalence between the Wiener-Hopf equation (2.5) and the generalized variational inequality which is to find $u \in K$ such that

$$
\langle T u+w, v-u\rangle \geq 0, \quad \forall v \in C, \forall w \in M u .
$$

Next, denote the sets of solutions of $(2.5)$ by $\operatorname{WHE}(T, S)$.

Lemma 2.7 (see [29]) The variational inequality (2.6) has a solution $\tilde{c} \in H$ if and only if the Wiener-Hopf equation (2.5) has a solution $\tilde{z} \in H$, where $\tilde{z}=\tilde{c}-\rho(T \tilde{c}+w), \tilde{c}=S P_{K} \tilde{z}$.

Lemma 2.8 (see [30]) Assume that $\left\{a_{n}\right\}$ is a sequence of nonnegative real numbers such that

$$
a_{n+1} \leq\left(1-\lambda_{n}\right) a_{n}+b_{n}, \quad \forall n \geq n_{0},
$$

where $n_{0}$ is some nonnegative integer, and $\left\{\lambda_{n}\right\}$ is a sequence in $[0,1]$ such that $\sum_{n=1}^{\infty} \lambda_{n}=\infty$, $b_{n}=o\left(\lambda_{n}\right)$. Then $\lim _{n \rightarrow \infty} a_{n}=0$.

Lemma 2.9 For given $x, z \in K$, if

$$
\langle y-z, z-x\rangle \geq 0
$$

for any $y \in K$. Then $x=z$.

Proof Assume $x \neq z$. Put $y=x$, then (2.7) reduces to $0 \leq\langle x-z, z-x\rangle=-\|x-z\|^{2}<0$, which is a contradiction.

\section{Results for solving $\operatorname{EP}(f) \cap \operatorname{VI}(K, T)$}

In this section, we firstly use Lemma 2.4 to introduce some iterative schemes and the convergence theorems for solving $\mathrm{EP}(f) \cap \mathrm{VI}(K, T)$. 
Algorithm 3.1 For a given $z_{0}$, compute the approximate solution $z_{n+1}$ by the iterative schemes:

$$
\left\{\begin{array}{l}
u_{n}=P_{K} z_{n}, \\
f\left(v_{n}, y\right)+\frac{1}{r}\left\langle y-v_{n}, v_{n}-u_{n}\right\rangle \geq 0, \quad \forall y \in K, \\
z_{n+1}=v_{n}-\rho T v_{n} .
\end{array}\right.
$$

By an appropriate rearrangement, Algorithm 3.1 can be written in the following form.

Algorithm 3.2 For a given $z_{0}$, compute the approximate solution $z_{n+1}$ by the iterative schemes:

$$
\left\{\begin{array}{l}
f\left(v_{n}, y\right)+\frac{1}{r}\left\langle y-v_{n}, v_{n}-P_{K} z_{n}\right\rangle \geq 0, \quad \forall y \in K, \\
z_{n+1}=v_{n}-\rho T v_{n} .
\end{array}\right.
$$

If $f(x, y)=0$ for all $x, y \in K$, Algorithm 3.1 collapses to the following iterative method for solving variational inequalities (1.2), which is mainly due to Shi [3].

Algorithm 3.3 For a given $z_{0}$, compute the approximate solution $z_{n+1}$ by the iterative schemes:

$$
\left\{\begin{array}{l}
u_{n}=P_{K} z_{n} \\
z_{n+1}=u_{n}-\rho T u_{n}
\end{array}\right.
$$

Theorem 3.1 Let $K$ be the nonempty closed convex subset of $H$. The bifunction $f: K \times K \rightarrow$ $R$ satisfies the conditions (i)-(iv) of Lemma 2.1. Let $T: K \rightarrow K$ be a $\alpha$-strongly monotone and $\beta$-Lipschitz continuous operator such that $\mathrm{EP}(f) \cap \operatorname{VI}(K, T) \neq \emptyset$. Let $\left\{z_{n}\right\},\left\{u_{n}\right\},\left\{v_{n}\right\}$ be the sequences generated by Algorithm 3.1, where $\rho>0$ is a constant and

$$
0<1-2 \alpha \rho+\beta^{2} \rho^{2}<1 .
$$

Then $\left\{u_{n}\right\},\left\{v_{n}\right\}$ generated by Algorithm 3.1 converge to $s \in \mathrm{EP}(f) \cap \mathrm{VI}(K, T)$, and $\left\{z_{n}\right\}$ generated by Algorithm 3.1 converges to $\tilde{z} \in \mathrm{WHE}(T)$.

Proof Let $\tilde{z} \in \mathrm{WHE}(T)$ and $s \in \mathrm{EP}(f) \cap \operatorname{VI}(K, T)$.

Step 1. Estimate $\left\|z_{n+1}-\tilde{z}\right\|$.

From Lemma 2.4, we have

$$
\begin{aligned}
& \tilde{z}=s-\rho T s, \\
& s=P_{K} \tilde{z} .
\end{aligned}
$$

Hence

$$
\begin{aligned}
\left\|z_{n+1}-\tilde{z}\right\| & =\left\|v_{n}-\rho T v_{n}-s+\rho T s\right\| \\
& =\sqrt{\left\|v_{n}-s-\left(\rho T v_{n}-\rho T s\right)\right\|^{2}}
\end{aligned}
$$




$$
\begin{aligned}
& \leq \sqrt{\left\|v_{n}-s\right\|^{2}-2 \rho\left\langle T v_{n}-T s, v_{n}-s\right\rangle+\left\|T v_{n}-T s\right\|^{2}} \\
& \leq \sqrt{1-2 \alpha \rho+\beta^{2} \rho^{2}}\left\|v_{n}-s\right\| .
\end{aligned}
$$

Here, we have used the $\alpha$-strong monotonicity and $\mu$-Lipschitz continuity of $T$ in (3.3) and (3.4).

Step 2. Estimate $\left\|v_{n}-s\right\|$.

Since $s \in \operatorname{EP}(f) \cap \operatorname{VI}(K, T)$, we have

$$
f(s, y) \geq 0, \quad \forall y \in K
$$

Putting $y=v_{n}$ in (3.5) and $y=s$ in Algorithm 3.1, we have

$$
f\left(s, v_{n}\right) \geq 0 \text { and } f\left(v_{n}, s\right)+\frac{1}{r}\left\langle s-v_{n}, v_{n}-u_{n}\right\rangle \geq 0
$$

From the monotonicity of $f$, we get

$$
f\left(s, v_{n}\right) \geq 0 \Rightarrow f\left(v_{n}, s\right) \leq 0 .
$$

Combining (3.6) and (3.7), we obtain

$$
\left\langle s-v_{n}, v_{n}-u_{n}\right\rangle \geq 0 .
$$

It follows that

$$
\begin{aligned}
& \left\langle s-v_{n}, v_{n}-s+s-u_{n}\right\rangle \geq 0 \\
& \Rightarrow \quad\left\langle s-v_{n}, v_{n}-s\right\rangle+\left\langle s-v_{n}, s-u_{n}\right\rangle \geq 0 \\
& \Rightarrow \quad\left\|s-v_{n}\right\|^{2} \leq\left\langle s-v_{n}, s-u_{n}\right\rangle \leq\left\|s-v_{n}\right\| \cdot\left\|s-u_{n}\right\| \\
& \Rightarrow \quad\left\|s-v_{n}\right\| \leq\left\|s-u_{n}\right\| \\
& \Rightarrow \quad\left\|v_{n}-s\right\| \leq\left\|u_{n}-s\right\| .
\end{aligned}
$$

Step 3. Estimate $\left\|u_{n}-s\right\|$ and prove the strong convergence of sequences generated by Algorithm 3.1.

Due to the nonexpansivity of $P_{K}$, we find

$$
\left\|u_{n}-s\right\|=\left\|P_{K} z_{n}-P_{K} \tilde{z}\right\| \leq\left\|z_{n}-\tilde{z}\right\|
$$

By the above three steps, we have

$$
\begin{aligned}
\left\|z_{n+1}-\tilde{z}\right\| & \leq \sqrt{\left(1-2 \alpha \rho+\beta^{2} \rho^{2}\right)}\left\|v_{n}-s\right\| \\
& \leq \sqrt{\left(1-2 \alpha \rho+\beta^{2} \rho^{2}\right)}\left\|u_{n}-s\right\| \\
& \leq \sqrt{\left(1-2 \alpha \rho+\beta^{2} \rho^{2}\right)}\left\|z_{n}-\tilde{z}\right\|,
\end{aligned}
$$


which implies that

$$
\begin{aligned}
\left\|z_{n+1}-\tilde{z}\right\| & \leq \sqrt{\left(1-2 \alpha \rho+\beta^{2} \rho^{2}\right)}\left\|z_{n}-\tilde{z}\right\| \leq \cdots \\
& \leq\left(\sqrt{\left(1-2 \alpha \rho+\beta^{2} \rho^{2}\right)}\right)^{n+1}\left\|z_{0}-\tilde{z}\right\| .
\end{aligned}
$$

Since

$$
\sqrt{\left(1-2 \alpha \rho+\beta^{2} \rho^{2}\right)}<1
$$

we conclude

$$
\left\|z_{n+1}-\tilde{z}\right\| \rightarrow 0 \quad(n \rightarrow \infty)
$$

Therefore, from

$$
\left\|v_{n}-s\right\| \leq\left\|u_{n}-s\right\|=\left\|P_{K} z_{n}-P_{K} z\right\| \leq\left\|z_{n}-z\right\|,
$$

we have

$$
\begin{array}{ll}
\left\|v_{n}-s\right\| \rightarrow 0 & (n \rightarrow \infty), \\
\left\|u_{n}-s\right\| \rightarrow 0 & (n \rightarrow \infty) .
\end{array}
$$

\section{Results for solving $\mathrm{EP}(f) \cap \mathrm{VI}(K, T) \cap F(S)$}

In this section, applying Lemma 2.6, we consider a nonexpansive mapping and analyze several iterative schemes and convergence theorems for solving $\operatorname{EP}(f) \cap \operatorname{VI}(K, T) \cap F(S)$.

Algorithm 4.1 For a given $z_{0} \in H$, compute the approximate solution $z_{n+1}$ by the iterative schemes

$$
\left\{\begin{array}{l}
u_{n}=\alpha_{n} P_{C} z_{n}+\left(1-\alpha_{n}\right) S P_{C} z_{n}, \\
f\left(v_{n}, y\right)+\frac{1}{r}\left\langle y-v_{n}, v_{n}-u_{n}\right\rangle \geq 0, \quad \forall y \in K, \\
z_{n+1}=\left(1-\alpha_{n}\right) z_{n}+\alpha_{n}\left(v_{n}-\rho T v_{n}\right) .
\end{array}\right.
$$

For $S=I$, the identity operator, Algorithm 4.1 collapses to the following iterative method.

Algorithm 4.2 For a given $z_{0} \in H$, compute the approximate solution $z_{n+1}$ by the iterative schemes

$$
\left\{\begin{array}{l}
u_{n}=P_{C} z_{n}, \\
f\left(v_{n}, y\right)+\frac{1}{r}\left\langle y-v_{n}, v_{n}-u_{n}\right\rangle \geq 0, \quad \forall y \in K, \\
z_{n+1}=\left(1-\alpha_{n}\right) z_{n}+\alpha_{n}\left(v_{n}-\rho T v_{n}\right) .
\end{array}\right.
$$

For $\alpha_{n}=1, S=I$, the identity operator, Algorithm 4.1 collapses to the following iterative method. 
Algorithm 4.3 For a given $z_{0} \in H$, compute the approximate solution $z_{n+1}$ by the iterative schemes

$$
\left\{\begin{array}{l}
u_{n}=P_{C} z_{n}, \\
f\left(v_{n}, y\right)+\frac{1}{r}\left\langle y-v_{n}, v_{n}-u_{n}\right\rangle \geq 0, \quad \forall y \in K, \\
z_{n+1}=v_{n}-\rho T v_{n} .
\end{array}\right.
$$

For $f=0$ and via Lemma 2.9, Algorithm 4.1 reduces to the following iterative method for solving $\operatorname{VI}(K, T) \cap F(S)$.

Algorithm 4.4 For a given $z_{0} \in H$, compute the approximate solution $z_{n+1}$ by the iterative schemes

$$
\left\{\begin{array}{l}
u_{n}=\alpha_{n} P_{C} z_{n}+\left(1-\alpha_{n}\right) S P_{C} z_{n} \\
z_{n+1}=\left(1-\alpha_{n}\right) z_{n}+\alpha_{n}\left(u_{n}-\rho T u_{n}\right)
\end{array}\right.
$$

For $f=0, S=I, \alpha_{n}=1$, and via Lemma 2.9, Algorithm 4.1 reduces to the following iterative method for solving VIP.

Algorithm 4.5 For a given $z_{0} \in H$, compute the approximate solution $z_{n+1}$ by the iterative schemes

$$
\left\{\begin{array}{l}
u_{n}=P_{C} z_{n} \\
z_{n+1}=u_{n}-\rho T u_{n}
\end{array}\right.
$$

Theorem 4.1 Let $K$ be the nonempty closed convex subset of $H$. The bifunction $f$ satisfies the conditions (i)-(iv) of Lemma 2.1. Let $T: K \rightarrow K$ be relaxed $(\gamma, r)$-co-coercive and $\mu$-Lipschitz continuous, and let $S: K \rightarrow K$ be a $k$-strictly pseudocontractive mapping such that $\operatorname{EP}(f) \cap \operatorname{VIP}(K, T) \cap F(S) \neq \emptyset$, where

$$
\sum_{n=0}^{\infty} \alpha_{n}=\infty, \quad \alpha \in[k, 1), \quad 0<\rho<\frac{2(r-\gamma \mu-k)}{(\mu+m)^{2}}, \quad r>\gamma \mu+k .
$$

Then the sequences $\left\{u_{n}\right\},\left\{v_{n}\right\}$ generated by Algorithm 4.1 converge to $\tilde{c} \in \mathrm{EP}(f) \cap \operatorname{VI}(K, T) \cap$ $F(S)$ and the sequence $\left\{z_{n}\right\}$ generated by Algorithm 4.1 converges to $\tilde{z} \in \operatorname{WHE}(T)$.

Proof Let $\tilde{z} \in \operatorname{WHE}(T)$ and $\tilde{c} \in \operatorname{EP}(f) \cap \operatorname{VI}(K, T) \cap F(S)$.

Step 1. Estimate $\left\|z_{n+1}-\tilde{z}\right\|$.

From Lemma 2.6, we have

$$
\begin{aligned}
& \tilde{z}=\tilde{c}-\rho T \tilde{c}, \\
& \tilde{c}=S P_{K} \tilde{z} .
\end{aligned}
$$

This implies that

$$
\begin{aligned}
& \tilde{c}=\alpha_{n} P_{C} \tilde{z}+\left(1-\alpha_{n}\right) S P_{C} \tilde{z}, \\
& \tilde{z}=\left(1-\alpha_{n}\right) \tilde{z}+\alpha_{n}(\tilde{c}-\rho T \tilde{c}) .
\end{aligned}
$$


Hence

$$
\begin{aligned}
\left\|z_{n+1}-\tilde{z}\right\| & \\
= & \left\|\left(1-\alpha_{n}\right) z_{n}+\alpha_{n}\left(v_{n}-\rho T v_{n}\right)-\tilde{z}\right\| \\
= & \left\|\left(1-\alpha_{n}\right) z_{n}+\alpha_{n}\left(v_{n}-\rho T v_{n}\right)-\left(1-\alpha_{n}\right) \tilde{z}-\alpha_{n}(\tilde{c}-\rho T \tilde{c})\right\| \\
= & \left\|\left(1-\alpha_{n}\right) z_{n}-\left(1-\alpha_{n}\right) \tilde{z}+\alpha_{n}\left(v_{n}-\rho T v_{n}\right)-\alpha_{n}(\tilde{c}-\rho T \tilde{c})\right\| \\
\leq & \left(1-\alpha_{n}\right)\left\|z_{n}-\tilde{z}\right\|+\alpha_{n}\left\|v_{n}-\tilde{c}-\rho\left(T v_{n}-T \tilde{c}\right)\right\| \\
= & \left(1-\alpha_{n}\right)\left\|z_{n}-\tilde{z}\right\|+\alpha_{n} \sqrt{\left\|v_{n}-\tilde{c}-\rho\left(T v_{n}-T \tilde{c}\right)\right\|^{2}} \\
= & \left(1-\alpha_{n}\right)\left\|z_{n}-\tilde{z}\right\|+\alpha_{n} \sqrt{\left\|v_{n}-\tilde{c}\right\|^{2}-2 \rho\left\langle T v_{n}-\rho T \tilde{c}, v_{n}-\tilde{c}\right\rangle+\rho^{2}\left\|T v_{n}-T \tilde{c}\right\|^{2}} \\
\leq & \left(1-\alpha_{n}\right)\left\|z_{n}-\tilde{z}\right\| \\
& +\alpha_{n} \sqrt{\left\|v_{n}-\tilde{c}\right\|^{2}-2 \rho\left[-\gamma\left\|T v_{n}-\rho T \tilde{c}\right\|^{2}+r\left\|v_{n}-\tilde{c}\right\|^{2}\right]+\rho^{2}\left\|T v_{n}-\rho T \tilde{c}\right\|^{2}} \\
\leq & \left(1-\alpha_{n}\right)\left\|z_{n}-\tilde{z}\right\| \\
& +\alpha_{n} \sqrt{\left\|v_{n}-\tilde{c}\right\|^{2}-2 \rho\left[-\gamma \mu^{2}\left\|v_{n}-\tilde{c}\right\|^{2}+r\left\|v_{n}-\tilde{c}\right\|^{2}\right]+\rho^{2} \mu^{2}\left\|v_{n}-\tilde{c}\right\|^{2}} \\
= & \left(1-\alpha_{n}\right)\left\|z_{n}-\tilde{z}\right\|+\alpha_{n} \sqrt{\left(1+2 \rho \gamma \mu^{2}-2 \rho r+\rho^{2} \mu^{2}\right)\left\|v_{n}-\tilde{c}\right\| .}
\end{aligned}
$$

In (4.8), (4.10), (4.11) of the above induction, we have used the $(\gamma, r)$-co-coercivity and $\mu$-Lipschitz continuity of the operator $T$.

Step 2. Estimate $\left\|v_{n}-\tilde{c}\right\|$.

Since $\tilde{c} \in \operatorname{EP}(f) \cap \operatorname{VI}(K, T) \cap F(S)$, we get

$$
f(\tilde{c}, y) \geq 0, \quad \forall y \in K
$$

Putting $y=v_{n}$ in (4.12) and $y=\tilde{c}$ in Algorithm 4.1, respectively, we have

$$
f\left(\tilde{c}, v_{n}\right) \geq 0 \quad \text { and } \quad f\left(v_{n}, \tilde{c}\right)+\frac{1}{r}\left\langle\tilde{c}-v_{n}, v_{n}-u_{n}\right\rangle \geq 0
$$

From the monotonicity of $f$, we obtain

$$
f\left(\tilde{c}, v_{n}\right) \geq 0 \Rightarrow f\left(v_{n}, \tilde{c}\right) \leq 0 .
$$

Combining (4.13) and (4.14), we know

$$
\left\langle\tilde{c}-v_{n}, v_{n}-u_{n}\right\rangle \geq 0 .
$$

It follows that

$$
\begin{aligned}
& \left\langle\tilde{c}-v_{n}, v_{n}-\tilde{c}+\tilde{c}-u_{n}\right\rangle \\
& \Rightarrow \quad\left\langle\tilde{c}-v_{n}, v_{n}-\tilde{c}\right\rangle+\left\langle\tilde{c}-v_{n}, \tilde{c}-u_{n}\right\rangle \\
& \Rightarrow \quad\left\|\tilde{c}-v_{n}\right\|^{2} \leq\left\langle\tilde{c}-v_{n}, \tilde{c}-u_{n}\right\rangle \leq\left\|\tilde{c}-v_{n}\right\| \cdot\left\|\tilde{c}-u_{n}\right\|
\end{aligned}
$$




$$
\begin{array}{ll}
\Rightarrow & \left\|\tilde{c}-v_{n}\right\| \leq\left\|\tilde{c}-u_{n}\right\| \\
\Rightarrow & \left\|v_{n}-\tilde{c}\right\| \leq\left\|u_{n}-\tilde{c}\right\| .
\end{array}
$$

Step 3. Estimate $\left\|u_{n}-\tilde{c}\right\|$ and prove the strong convergence of sequences generated by Algorithm 4.1.

Since

$$
\begin{aligned}
& u_{n}=\alpha_{n} P_{C} z_{n}+\left(1-\alpha_{n}\right) S P_{C} z_{n}, \\
& \tilde{c}=\alpha_{n} P_{C} \tilde{z}+\left(1-\alpha_{n}\right) S P_{C} \tilde{z},
\end{aligned}
$$

we have

$$
\begin{aligned}
\left\|u_{n}-\tilde{c}\right\| & =\left\|\alpha_{n} P_{C} z_{n}+\left(1-\alpha_{n}\right) S P_{C} z_{n}-\alpha_{n} P_{C} \tilde{z}-\left(1-\alpha_{n}\right) S P_{C} \tilde{z}\right\| \\
& =\left\|\alpha_{n} P_{C} z_{n}-\alpha_{n} P_{C} \tilde{z}+\left(1-\alpha_{n}\right) S P_{C} z_{n}-\left(1-\alpha_{n}\right) S P_{C} \tilde{z}\right\| \\
& \leq \alpha_{n}\left\|P_{C} z_{n}-P_{C} \tilde{z}\right\|+\left(1-\alpha_{n}\right)\left\|S P_{C} z_{n}-S P_{C} \tilde{z}\right\| \\
& \leq\left\|z_{n}-\tilde{z}\right\| .
\end{aligned}
$$

By the above three steps, we get

$$
\begin{aligned}
\left\|z_{n+1}-\tilde{z}\right\| & \leq\left(1-\alpha_{n}\right)\left\|z_{n}-\tilde{z}\right\|+\alpha_{n} \sqrt{\left(1+2 \rho \gamma \mu^{2}-2 \rho r+\rho^{2} \mu^{2}\right)}\left\|v_{n}-\tilde{c}\right\| \\
& \leq\left(1-\alpha_{n}\right)\left\|z_{n}-\tilde{z}\right\|+\alpha_{n} \sqrt{\left(1+2 \rho \gamma \mu^{2}-2 \rho r+\rho^{2} \mu^{2}\right)}\left\|u_{n}-\tilde{c}\right\| \\
& \leq\left(1-\alpha_{n}\right)\left\|z_{n}-\tilde{z}\right\|+\alpha_{n} \sqrt{\left(1+2 \rho \gamma \mu^{2}-2 \rho r+\rho^{2} \mu^{2}\right)}\left\|z_{n}-\tilde{c}\right\| \\
& =\left[1-\alpha_{n}(1-\theta)\right]\left\|z_{n}-\tilde{c}\right\|,
\end{aligned}
$$

where $\theta=\sqrt{\left(1+2 \rho \gamma \mu^{2}-2 \rho r+\rho^{2} \mu^{2}\right)}$.

From Lemma 2.8, it is easy to see that

$$
\left\|z_{n}-\tilde{z}\right\| \rightarrow 0 \quad(n \rightarrow \infty)
$$

and from

$$
\left\|v_{n}-s\right\| \leq\left\|u_{n}-s\right\|=\left\|P_{K} z_{n}-P_{K} z\right\| \leq\left\|z_{n}-z\right\|
$$

we have

$$
\begin{array}{ll}
\left\|u_{n}-s\right\| \rightarrow 0 & (n \rightarrow \infty), \\
\left\|v_{n}-s\right\| \rightarrow 0 & (n \rightarrow \infty) .
\end{array}
$$

\section{Generation}

Ever since the classical variational inequality was introduced, it has been extended to many forms in different directions, such as the nonconvex variational inequality, the multivalued variational inequality, the extended general quasi-variational inequalities and so 
on. Related to the variational inequality, the Wiener-Hopf equation has also been extended, and the equivalence between generalized variational inequalities and generalized Wiener-Hopf equations has been studied.

In this section, we consider the two generalized variational inequalities (2.3) and (2.6), respectively. Furthermore, applying Lemma 2.5 , we firstly suggest and give some iterative schemes for solving the variational inequality (2.3) and the equilibrium problem; secondly, via Lemma 2.7, we also analyze and introduce several iterative schemes for solving the variational inequality (2.6), the equilibrium problem, and a nonexpansive mapping.

\subsection{Results for solving variational inequality (2.3) and equilibrium problem}

In this subsection, we use a similar technique to Section 3 , and the proposed results in this subsection can be considered as the generation of Section 3 .

Algorithm 5.1 For a given $z_{0} \in H$, compute the approximate solution $z_{n+1}$ by the iterative schemes

$$
\left\{\begin{array}{l}
g\left(u_{n}\right)=P_{K} z_{n}, \\
f\left(v_{n}, y\right)+\frac{1}{r}\left\langle y-v_{n}, v_{n}-u_{n}\right\rangle \geq 0, \quad \forall y \in K, \\
z_{n+1}=g\left(v_{n}\right)-\rho T v_{n} .
\end{array}\right.
$$

Theorem 5.1 Let $K$ be the nonempty closed convex subset of $H$ and the bifunction $f$ : $K \times K \rightarrow R$ satisfy the conditions (i)-(iv) of Lemma 2.1. Let $T: K \rightarrow K$ be a $\alpha$-strongly monotone and $\beta$-Lipschitz continuous single-valued operator and let $g: K \rightarrow K$ be a $\sigma$-strongly monotone and $\delta$-Lipschitz continuous single-valued operator such that $g^{-1}$ exists and $\mathrm{EP}(f) \cap \operatorname{GVI}(K, T) \neq \emptyset$, where

$$
k=\sqrt{1-2 \sigma+\delta^{2}} \neq 1, \quad t=\sqrt{1-2 \rho \alpha+\beta^{2} \rho^{2}}, \quad 0<\frac{k+t}{1-k}<1, \quad \rho>0 .
$$

Then the sequences $\left\{u_{n}\right\},\left\{v_{n}\right\}$ generated by Algorithm 5.1 converge strongly to $s \in \operatorname{EP}(f) \cap$ $\operatorname{GVI}(K, T)$ and $\left\{z_{n}\right\}$ generated by Algorithm 5.1 converges strongly to $\tilde{z} \in \operatorname{WHE}(T, g)$.

Proof Let $s \in \mathrm{EP}(f) \cap \operatorname{GVI}(K, T), \tilde{z} \in \operatorname{WHE}(T, g)$.

Step 1. Estimate $\left\|z_{n+1}-\tilde{z}\right\|$.

From the Lemma 2.5, we have

$$
\begin{aligned}
& g(s)=P_{K} \tilde{z}, \\
& \tilde{z}=g(s)-\rho T s .
\end{aligned}
$$

This implies that

$$
\begin{aligned}
\left\|z_{n+1}-\tilde{z}\right\|= & \left\|g\left(v_{n}\right)-g(s)-\rho\left(T v_{n}-T s\right)\right\| \\
\leq & \left\|v_{n}-s-\left(g\left(v_{n}\right)-g(s)\right)\right\|+\left\|v_{n}-s-\rho\left(T v_{n}-T s\right)\right\| \\
= & \sqrt{\left\|v_{n}-s\right\|^{2}-2\left\langle g\left(v_{n}\right)-g(s), v_{n}-s\right\rangle+\left\|g\left(v_{n}\right)-g(s)\right\|^{2}} \\
& +\sqrt{\left\|v_{n}-s\right\|^{2}-2 \rho\left\langle T v_{n}-T s, v_{n}-s\right\rangle+\rho^{2}\left\|T v_{n}-T s\right\|^{2}}
\end{aligned}
$$




$$
\begin{aligned}
& \leq \sqrt{1-2 \sigma+\delta^{2}}\left\|v_{n}-s\right\|+\sqrt{1-2 \rho \alpha+\beta^{2} \rho^{2}}\left\|v_{n}-s\right\| \\
& \leq(k+t)\left\|v_{n}-s\right\|^{2} .
\end{aligned}
$$

Note that we use the strong monotonicity and Lipschitz continuity of $T, g$ in (5.8), where $k=\sqrt{1-2 \sigma+\delta^{2}} \neq 1, t=\sqrt{1-2 \rho \alpha+\beta^{2} \rho^{2}}$.

Step 2. Estimate $\left\|v_{n}-s\right\|$.

Employing the technique that we use in the proof of Theorem 3.1, we get

$$
\left\|v_{n}-s\right\| \leq\left\|u_{n}-s\right\|
$$

Step 3. Estimate $\left\|u_{n}-s\right\|$ and prove the strong convergence of sequences generated by Algorithm 5.1.

From (5.3), we obtain

$$
\begin{aligned}
\left\|u_{n}-s\right\| & \leq\left\|u_{n}-s-\left(g\left(u_{n}\right)-g(s)\right)+P_{K} z_{n}-P_{K} z\right\| \\
& \leq k\left\|u_{n}-s\right\|+\left\|z_{n}-z\right\| .
\end{aligned}
$$

It follows that

$$
\left\|u_{n}-s\right\| \leq\left(\frac{1}{1-k}\right)\left\|z_{n}-z\right\| \quad(k \neq 1) .
$$

Combining the above three steps, we have

$$
\begin{aligned}
\left\|z_{n+1}-z\right\| & \leq(k+t)\left\|v_{n}-s\right\| \leq(k+t)\left\|u_{n}-s\right\| \\
& \leq\left(\frac{k+t}{1-k}\right)\left\|z_{n}-z\right\| .
\end{aligned}
$$

Since

$$
0<\frac{k+t}{1-k}<1
$$

we get

$$
\begin{array}{ll}
\left\|z_{n}-\tilde{z}\right\| \rightarrow 0 & (n \rightarrow \infty), \\
\left\|u_{n}-s\right\| \rightarrow 0 & (n \rightarrow \infty), \\
\left\|v_{n}-s\right\| \rightarrow 0 & (n \rightarrow \infty) .
\end{array}
$$

Remark 5.1 Clearly, if $g$ is the identity mapping, Theorem 5.1 reduces to Theorem 3.1.

Remark 5.2 Via Lemma 2.9, it is easy to see that if the bifunction $f(x, y)=0, \forall x, y \in K$, Algorithm 5.1 reduces to

$$
\left\{\begin{array}{l}
g\left(u_{n}\right)=P_{K} z_{n}, \\
z_{n+1}=g\left(u_{n}\right)-\rho T u_{n} .
\end{array}\right.
$$

This algorithm was introduced by Noor in [4], which implies that Algorithm 5.1 in this paper extends the results in [4]. 
Remark 5.3 It is easy to show the condition (5.1) can be satisfied, for instance

$$
\alpha=\frac{7}{4}, \quad \beta=\sqrt{2}, \quad \delta=\frac{\sqrt{2}}{10}, \quad \sigma=\frac{49}{100}, \quad \rho=\frac{1}{4} .
$$

\subsection{Results for solving variational inequality (2.6), an equilibrium problem, and a nonexpansive mapping}

In this subsection, we use a similar technique to Section 4, and the proposed results in this subsection can be considered as the generation of Section 4 .

Algorithm 5.2 For a given $z_{0} \in H$, compute the approximate solution $z_{n+1}$ by the iterative schemes

$$
\left\{\begin{array}{l}
u_{n}=\alpha P_{C} z_{n}+(1-\alpha) S P_{C} z_{n}, \\
f\left(v_{n}, y\right)+\frac{1}{r}\left\langle y-v_{n}, v_{n}-u_{n}\right\rangle \geq 0, \quad \forall y \in K, \\
z_{n+1}=\left(1-\alpha_{n}\right) z_{n}+\alpha_{n}\left[v_{n}-\rho\left(T v_{n}+w_{n}\right)\right] .
\end{array}\right.
$$

If $S=I$, Algorithm 5.2 reduces to the following.

Algorithm 5.3 For a given $z_{0} \in H$, compute the approximate solution $z_{n+1}$ by the iterative schemes

$$
\left\{\begin{array}{l}
u_{n}=P_{C} z_{n}, \\
f\left(v_{n}, y\right)+\frac{1}{r}\left\langle y-v_{n}, v_{n}-u_{n}\right\rangle \geq 0, \quad \forall y \in K, \\
z_{n+1}=\left(1-\alpha_{n}\right) z_{n}+\alpha_{n}\left[v_{n}-\rho\left(T v_{n}+w_{n}\right)\right] .
\end{array}\right.
$$

If $S=I$ and $\alpha_{n}=1$, Algorithm 5.2 reduces to the following.

Algorithm 5.4 For a given $z_{0} \in H$, compute the approximate solution $z_{n+1}$ by the iterative schemes

$$
\left\{\begin{array}{l}
u_{n}=P_{C} z_{n}, \\
f\left(v_{n}, y\right)+\frac{1}{r}\left\langle y-v_{n}, v_{n}-u_{n}\right\rangle \geq 0, \quad \forall y \in K, \\
z_{n+1}=v_{n}-\rho\left(T v_{n}+w_{n}\right) .
\end{array}\right.
$$

If $f=0$, Algorithm 5.2 reduces to the following.

Algorithm 5.5 For a given $z_{0} \in H$, compute the approximate solution $z_{n+1}$ by the iterative schemes

$$
\left\{\begin{array}{l}
u_{n}=\alpha P_{C} z_{n}+(1-\alpha) S P_{C} z_{n}, \\
z_{n+1}=\left(1-\alpha_{n}\right) z_{n}+\alpha_{n}\left[v_{n}-\rho\left(T v_{n}+w_{n}\right)\right],
\end{array}\right.
$$

which was introduced in [8].

If $f=0, S=I$, Algorithm 5.2 reduces to the following. 
Algorithm 5.6 For a given $z_{0} \in H$, compute the approximate solution $z_{n+1}$ by the iterative schemes

$$
\left\{\begin{array}{l}
u_{n}=S P_{C} z_{n}, \\
z_{n+1}=\left(1-\alpha_{n}\right) z_{n}+\alpha_{n}\left[v_{n}-\rho\left(T v_{n}+w_{n}\right)\right],
\end{array}\right.
$$

which was introduced in [8].

If $f=0, S=I$, and $\alpha_{n}=1$, Algorithm 5.2 reduces to the following.

Algorithm 5.7 For a given $z_{0} \in H$, compute the approximate solution $z_{n+1}$ by the iterative schemes

$$
\left\{\begin{array}{l}
u_{n}=S P_{C} z_{n}, \\
z_{n+1}=u_{n}-\rho\left(T u_{n}+w_{n}\right),
\end{array}\right.
$$

which was introduced in [6].

Theorem 5.2 Let $K$ be the nonempty closed convex subset of $H$ and the bifunction $f$ satisfy the conditions (i)-(iv) of Lemma 2.1. Let $T: K \rightarrow K$ be a relaxed $(\gamma, r)$-co-coercive and $\mu$-Lipschitz continuous operator, and $S: K \rightarrow K$ be k-strictly such that $\operatorname{EP}(f) \cap \operatorname{VI}(K, T) \cap$ $F(S) \neq \emptyset$. Let $M: H \rightarrow 2^{H}$ be a multi-valued relaxed monotone and Lipschitz continuous operator with the corresponding constant $k>0, m>0$, where

$$
\sum_{n=0}^{\infty} \alpha_{n}=\infty, \quad \alpha \in[k, 1), \quad 0<\rho<\frac{2(r-\gamma \mu-k)}{(\mu+m)^{2}}, \quad r>\gamma \mu+k .
$$

Then the sequences $\left\{u_{n}\right\},\left\{v_{n}\right\}$ generated by Algorithm 5.2 converge strongly to $\tilde{c} \in \operatorname{EP}(f) \cap$ $\operatorname{VI}(K, T) \cap F(S)$, and $\left\{z_{n}\right\}$ generated by Algorithm 5.2 converges strongly to $\tilde{z} \in \operatorname{WHE}(T, S)$.

Proof Let $\tilde{c} \in \operatorname{EP}(f) \cap \operatorname{VI}(K, T) \cap F(S)$ and $\tilde{z} \in \operatorname{WHE}(T, S)$.

Step 1. Estimate $\left\|z_{n+1}-\tilde{z}\right\|$ and $\left\|u_{n}-\tilde{c}\right\|$.

By the same technique in [29], we have

$$
\begin{aligned}
& \left\|z_{n+1}-\tilde{z}\right\| \leq\left(1-\alpha_{n}\right)\left\|z_{n}-\tilde{z}\right\|+\alpha_{n} \theta\left\|v_{n}-\tilde{c}\right\|, \\
& \left\|u_{n}-\tilde{c}\right\| \leq\left\|z_{n}-\tilde{z}\right\|,
\end{aligned}
$$

where $\theta=\sqrt{1+2 \rho(\gamma \mu-r+k)+\rho^{2}(\mu+m)^{2}}$.

Step 2. Estimate $\left\|v_{n}-\tilde{c}\right\|$.

Applying the technique in Theorem 3.2 of this paper, we get

$$
\left\|v_{n}-\tilde{c}\right\| \leq\left\|u_{n}-\tilde{c}\right\| .
$$

Combining the above two steps, we can obtain

$$
\begin{aligned}
\left\|z_{n+1}-\tilde{z}\right\| & \leq\left(1-\alpha_{n}\right)\left\|z_{n}-\tilde{z}\right\|+\alpha \theta\left\|u_{n}-\tilde{c}\right\| \\
& \leq\left(1-\alpha_{n}\right)\left\|z_{n}-\tilde{z}\right\|+\alpha \theta\left\|z_{n}-\tilde{c}\right\| \\
& =\left[1-\alpha_{n}(1-\theta)\right]\left\|z_{n}-\tilde{z}\right\| .
\end{aligned}
$$


From Lemma 2.8, it follows that

$$
\left\|z_{n}-\tilde{z}\right\| \rightarrow 0 \quad(n \rightarrow \infty)
$$

\section{Note that}

$$
\left\|v_{n}-s\right\| \leq\left\|u_{n}-s\right\| \leq\left\|z_{n}-z\right\|
$$

\section{We conclude that}

$$
\begin{array}{ll}
\left\|u_{n}-s\right\| \rightarrow 0 & (n \rightarrow \infty), \\
\left\|v_{n}-s\right\| \rightarrow 0 & (n \rightarrow \infty) .
\end{array}
$$

\section{Competing interests}

The authors declare that they have no competing interests.

\section{Authors' contributions}

All authors contributed equally to the writing of this paper. All authors read and approved the final manuscript.

\section{Author details}

${ }^{1}$ School of Mathematical Science, Anhui University, Hefei, 230601, P.R. China. ${ }^{2}$ School of Applied Mathematics, Nanjing University of Finance and Economics, Nanjing, 210023, P.R. China.

\section{Acknowledgements}

The authors would like to thank the referees for the valuable suggestions, which helped to improve this manuscript. This project is supported by the National Natural Science Foundation of China (11071109).

\section{Received: 7 March 2014 Accepted: 14 July 2014 Published: 18 Aug 2014}

\section{References}

1. Blum, E, Oettli, W: From optimization and variational inequalities to equilibrium problems. Math. Stud. 63, 123-145 (1994)

2. Stampacchia, G: Formes bilinéaires coercitives sur les ensembles convexes. C. R. Acad. Sci. Paris 258, 4413-4416 (1964)

3. Shi, P: Equivalence of variational inequalities with Wiener-Hopf equations. Proc. Am. Math. Soc. 111, $339-346$ (1991)

4. Noor, MA: Wiener-Hopf equations and variational inequalities. J. Optim. Theory Appl. 79(1), 197-206 (1993)

5. Noor, MA, Noor, Kl, Khan, AG: Some iterative schemes for solving extended general quasi-variational inequalities. Appl. Math. Inf. Sci. 7(3), 917-925 (2010)

6. Verma, RU: Generalized variational inequalities involving multivalued relaxed monotone operators. Appl. Math. Lett. 10, 107-109 (1997)

7. Al-Shemas, E: General nonconvex Wiener-Hopf equations and general nonconvex variational inequalities. J. Math. Sci. Adv. Appl. 19(1), 1-11 (2013)

8. Takahashi, S, Takahashi, W: Viscosity approximation methods for equilibrium problems and fixed point problems in Hilbert spaces. J. Math. Anal. Appl. 331, 506-515 (2007)

9. Li, H, Su, Y: Strong convergence theorem by a new hybrid method for equilibrium problems and variational inequality problems. Nonlinear Anal. 72, 847-855 (2010)

10. Plubtieng, S, Punpaeng, R: A general iterative method for equilibrium problems and fixed point problems in Hilbert spaces. J. Math. Anal. Appl. 336(1), 455-469 (2007)

11. Ansari, QH, Yao, JC: An existence result for the generalized vector equilibrium problem. Appl. Math. Lett. 12, 53-56 (1999)

12. Anh, LQ, Khanh, PQ: On the Hölder continuity of solutions to parametric multi-valued vector equilibrium problems. J. Math. Anal. Appl. 321, 308-315 (2006)

13. Bianchi, M, Kassay, G, Pini, R: Existence of equilibria via Ekeland's principle. J. Math. Anal. Appl. 305, 502-512 (2005)

14. Bianchi, M, Pini, R: A note on stability for parametric equilibrium problems. Oper. Res. Lett. 31, 445-450 (2003)

15. Cubiotti, P: Existence of solutions for lower semicontinuous quasi-equilibrium problems. Comput. Math. Appl. 30(12), 11-22 (1995)

16. Halpern, B: Fixed point of nonexpansive maps. Bull. Am. Math. Soc. 73, 957-961 (1967)

17. Ishikawa, S: Fixed points by a new iteration method. Proc. Am. Math. Soc. 44, 147-150 (1974)

18. Moudafi, A: Viscosity approximation methods for fixed-point problems. J. Math. Anal. Appl. 241, 46-55 (2000)

19. Noor, MA: General variational inequalities. Appl. Math. Lett. 1, 119-121 (1988)

20. Noor, MA: Multivalued general equilibrium problems. J. Math. Anal. Appl. 283, 140-149 (2003)

21. Noor, MA, Rassias, TM: On nonconvex equilibrium problems. J. Math. Anal. Appl. 312, 289-299 (2005)

22. Noor, MA: Iterative methods for general nonconvex variational inequalities. Albanian J. Math. 3(1), 117-127 (2009) 
23. Nakajo, K, Takahashi, W: Strong convergence theorems for nonexpansive mappings and nonexpansive semigroups. J. Math. Anal. Appl. 279, 372-379 (2003)

24. Qin, X, Noor, MA: General Wiener-Hopf equation technique for nonexpansive mappings and general variational inequalities in Hilbert spaces. Appl. Math. Comput. 201, 716-722 (2008)

25. Robinson, SM: Normal maps induced by linear transformations. Math. Oper. Res. 17, 691-714 (1992)

26. Glowinski, R, Lions, JL, Trémolières, R: Numerical Analysis of Variational Inequalities. North-Holland, Amsterdam (1981)

27. Su, Y, Shang, M, Qin, X: An iterative method of solution for equilibrium and optimization problems. Nonlinear Anal. 69, 2709-2719 (2008)

28. Takahashi, W, Toyoda, M: Weak convergence theorems for nonexpansive mappings and monotone mappings. J. Optim. Theory Appl. 118, 417-428 (2003)

29. Wu, C, Li, Y: Wiener-Hopf equations techniques for generalized variational inequalities and fixed point problems. In: 4th International Congress on Image and Signal Processing, pp. 2802-2805. IEEE Press, New York (2011)

30. Xu, HK: Viscosity approximation methods for nonexpansive mappings. J. Math. Anal. Appl. 298, 279-291 (2004)

31. Zhang, CJ: A class of equilibrium problems with lower and upper bounds. Acta Math. Sin. 48, $293-298$ (2005)

32. Noor, MA: On general quasi-variational inequalities. J. King Saud Univ., Sci. 24, 81-88 (2012)

33. Noor, MA, Huang, Z: Wiener-Hopf equation technique for variational inequalities and nonexpansive mappings. Appl. Math. Comput. 191, 504-510 (2007)

10.1186/1029-242X-2014-286

Cite this article as: Wang and Zhang: Wiener-Hopf equation technique for solving equilibrium problems and variational inequalities and fixed points of a nonexpansive mapping. Journal of Inequalities and Applications 2014, 2014:286

\section{Submit your manuscript to a SpringerOpen ${ }^{\circ}$ journal and benefit from:}

- Convenient online submission

- Rigorous peer review

- Immediate publication on acceptance

- Open access: articles freely available online

- High visibility within the field

- Retaining the copyright to your article 